Document downloaded from:

http://hdl.handle.net/10251/141938

This paper must be cited as:

Gomez Molina, C.; Quesada Pereira, F.; Alvarez Melcon, A.; Boria Esbert, VE.; Guglielmi, M. (02-2). An Efficient Technique to Assess the Convergence of the Multimode Equivalent Network for Waveguide Devices. IEEE Transactions on Microwave Theory and Techniques. 66(2):651-659. https://doi.org/10.1109/TMTT.2017.2785837

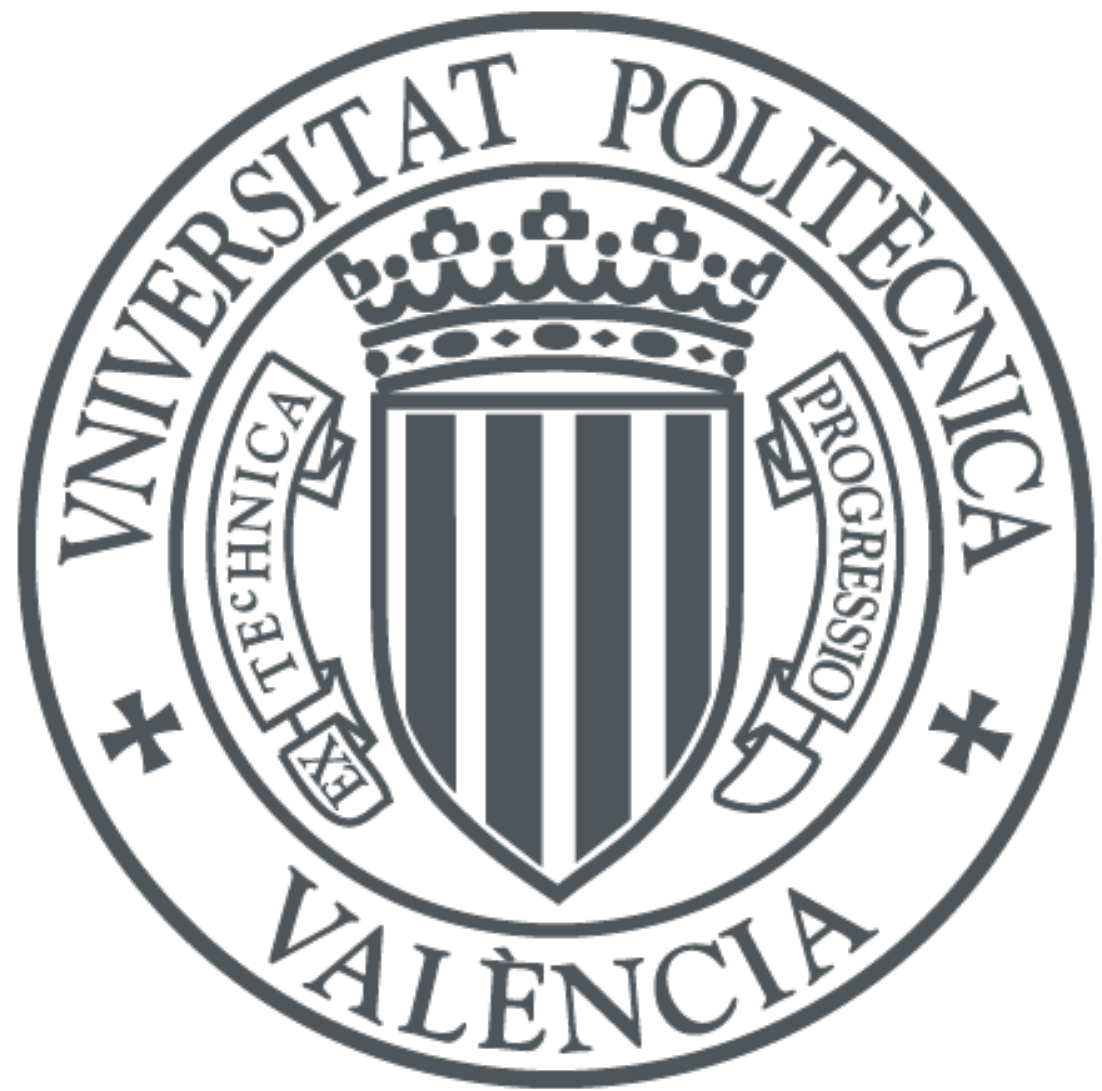

The final publication is available at

https://doi.org/10.1109/TMTT.2017.2785837

Copyright Institute of Electrical and Electronics Engineers

Additional Information 


\title{
An Efficient Technique to Assess the Convergence of the Multimode Equivalent Network for Waveguide Devices
}

\author{
Celia Gómez Molina, Fernando Quesada Pereira, Member, IEEE, \\ Alejandro Alvarez Melcón, Senior Member, IEEE, Vicente E. Boria, Fellow, IEEE, \\ and Marco Guglielmi, Fellow, IEEE
}

\begin{abstract}
Numerical methods are widely used to analyze and design microwave components for communication applications. In the implementation of any numerical technique, however, there are always a set of parameters that must be properly adjusted in order to obtain, at the same time, computational efficiency and numerical accuracy of the results. In this context, therefore, we focus in this paper on the multimode equivalent network formulation for waveguide devices, and we propose a more intuitive and efficient strategy for choosing these parameters. Following our approach, setting only one global numerical variable is sufficient to adjust automatically the specific convergence parameters of each discontinuity to give a specific level of numerical accuracy of the results. As a consequence, the computational efficiency is significantly increased. In addition, the user experience is significantly improved since our approach eliminates all lengthy convergence tests previously needed to assure good numerical accuracy. In addition to theory, we discuss in this paper a number of numerical results that clearly demonstrate how the new strategy is very effective, thereby fully validating the theoretical formulation.
\end{abstract}

Index Terms-Convergence, integral equations, method of moments (MoM), multimode equivalent networks, numerical methods, waveguide filters, waveguide junctions.

\section{INTRODUCTION}

$\mathbf{N}$ UMERICAL methods for solving electromagnetic (EM) problems are attracting more and more attention in recent years due to their ability of saving development time and

Manuscript received July 30, 2017; $\operatorname{xxxxxxxxxx}$ This work was supported in part by the Spanish Government through the Ministerio de Educación, Cultura y Deporte under Grant FPU15/02883, in part by the Ministerio de Economía y Competitividad through the sub-projects 4 and 1 of the coordinated project under Grant TEC2016-75934-C4-R, and in part by the Fundación Séneca de la Región de Murcia under Grant 19494/PI/14 and Grant 20147/EE/17. This paper is an expanded version from the IEEE MTT-S International Conference on Numerical Electromagnetic Modeling and Optimization for RF, Microwave, and Terahertz Applications, Seville, Spain, May 17-19, 2017. (Corresponding author: Celia Gómez Molina.)

C. Gómez Molina, F. Quesada Pereira, and A. Alvarez Melcón are with the Department of Information Technologies and Communications, Universidad Politécnica de Cartagena, 30202 Cartagena, Spain (e-mail: celia.gomez@upct.es; fernando.quesada@upct.es; alejandro.alvarez@upct.es).

V. E. Boria and M. Guglielmi are with the R\&D Institute on Telecommunication and Multimedia Applications, Universidad Politécnica de Valencia, 46022 Valencia, Spain (e-mail: vboria@dcom.upv.es; marco.guglielmi@iteam.upv.es).

Color versions of one or more of the figures in this paper are available online at http://ieeexplore.ieee.org. experimental work in real communication projects. In this context, therefore, a substantial research effort has been devoted to the development of fast and accurate EM models for the analysis and design of the needed microwave communication components. Furthermore, a continuous effort is also devoted to the reduction of the needed computational burden.

In this context, therefore, several EM analysis methods have been proposed in the technical literature. Examples are the finite element method [1], the finite differences in time domain [2], and others based on modal methods or integral equations [3], [4]. Commercial software tools, such as Ansoft HFSS, CST Studio, and FEST3D [5], for instance, implement some of these techniques, show good agreement with real measurements, and also exhibit good numerical efficiency.

In general, however, any analysis method must be eventually implemented numerically. As a consequence, there are always a set of numerical parameters that need to be properly adjusted in order to reach convergent results for the components under analysis. If the parameters are undersized, the response may not be convergent, that is, not accurate. On the other hand, if we increase the parameters more than what it is required, the problem may be oversized. This, in turn, may lead to an unnecessary increment of the computational effort, to ill conditioned situations, or even to relative convergence problems [6].

It is clear, therefore, that a careful selection of these numerical parameters is needed to find a compromise between accuracy and efficiency.

Currently, the correct convergence of the numerical methods that are integrated in commercial software tools continue to be a critical issue. From the user's point of view, this implies the responsibility of selecting the proper parameters, without the complete knowledge (in some cases) of the numerical methods being implemented. This process usually requires repeated simulations of the same structure with increased numerical parameters, until convergence is reached. The whole process can then be very time-consuming.

In this paper, we focus on the study of the convergence properties of the multimode equivalent network (MEN) formulation [7] for the analysis of waveguide components. This technique has indeed been proved to be fast and accurate for the analysis of many complex microwave devices [8]. The MEN technique is based on an integral equation formulation. In particular, the imposition of the boundary conditions at 


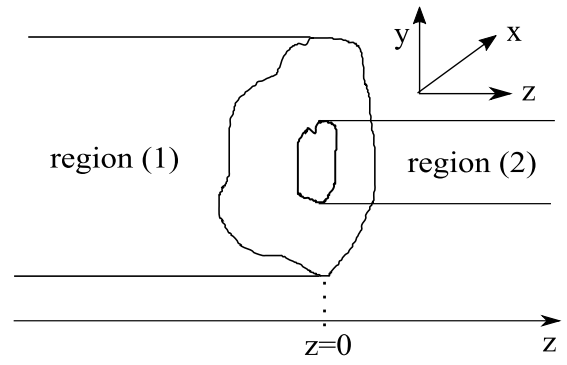

Fig. 1. 2-D step in waveguide technology.

the waveguide junctions leads to integral equations that are typically solved by the method of moments (MoM) [9]. The formulation finally produces an equivalent network, where the interactions between all higher order modes are rigorously accounted for by a generalized impedance or admittance multimode coupling matrix.

Furthermore, the numerical efficiency of the MEN approach has been recently enhanced by a novel formulation of the kernel of the cited integral equation [10]. There, the kernel is rigorously split into a dynamic and static parts. Thanks to this transformation; the number of modes that needs to be accessible in the equivalent network is no longer linked to the convergence of the kernel.

To further exploit this advantage, we propose, in this paper, a new strategy to select the numerical parameters that are needed to ensure convergence in the MEN formulation. In the remainder of this paper, we first discuss, in Section II, the novel strategy to select the numerical parameters for the MEN formulation. We then discuss, in Section III, a number of practical application examples clearly showing the improvements obtained, thereby fully validating the proposed strategy. Finally, the main conclusions of this paper are outlined in Section IV.

\section{New Convergence Method for MEN FORMULATION OF WAVEGUIDE DISCONTINUITIES}

In this section, we briefly review the general MEN formulation for 2-D waveguide junctions, as shown in Fig. 1. We then explain the new strategy to select the numerical parameters that ensure the accuracy of the computational results.

To start, we impose the continuity of the tangential component of the magnetic field $\mathbf{H}_{t}^{(\delta)}$ in the aperture at $z=0$. We write this condition using the well-known modal expansion formalism [7], [11] as follows:

$$
\begin{aligned}
& \sum_{n=1}^{N(1)} I_{n}^{(1)} \mathbf{h}_{n}^{(1)}(s)-\sum_{n=1}^{N(2)} I_{n}^{(2)} \mathbf{h}_{n}^{(2)}(s) \\
& =\sum_{m=N(1)+1}^{+\infty} Y_{m}^{(1)} V_{m}^{(1)} \mathbf{h}_{m}^{(1)}(s)+\sum_{m=N(2)+1}^{+\infty} Y_{m}^{(2)} V_{m}^{(2)} \mathbf{h}_{m}^{(2)}(s)
\end{aligned}
$$

where $\mathbf{h}_{m}^{(\delta)}(s)$ is the magnetic vector modal function of mode $m$ in the medium $(\delta), s$ indicates a point in the waveguide cross section, $I_{m}^{(\delta)}$ and $V_{m}^{(\delta)}$ represent the total modal currents and voltages, respectively, and $Y_{m}^{(\delta)}$ is the characteristic modal

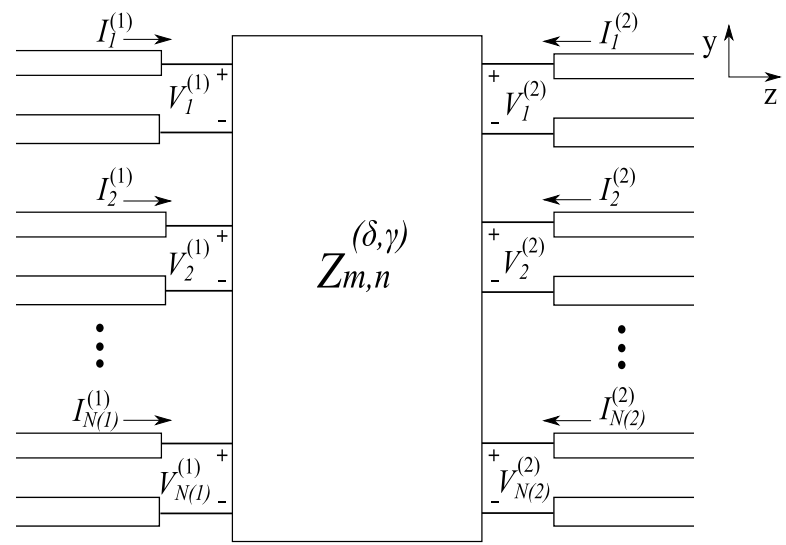

Fig. 2. MEN (generalized $Z$-matrix) representation for the waveguide step in Fig. 1.

admittance. In Fig. $1, \delta=1$ or $\delta=2$ for $z<0$ or $z>0$, respectively.

In (1), we have separated the accessible from localized modes by splitting the summation in $N(\delta)$ terms. Thus, the parameter $N(\delta)$ controls the number of accessible modes in the resulting equivalent network for the waveguide $(\delta)$. These modes are explicitly connected to the following discontinuity (another MEN as in Fig. 2) by lengths of transmission lines. The rest of the modes in the right-hand side of (1) are taken into account as localized ones in the kernel of the integral equation. Following the theory reported in [7] and [10], we can finally obtain the fundamental integral equation of our problem as

$$
\mathbf{h}_{n}^{(\delta)}(s)=\int_{a p} \mathbf{M}_{n}^{(\delta)}\left(s^{\prime}\right) \mathbf{K}\left(s, s^{\prime}\right) d s^{\prime}
$$

where $\mathbf{M}_{n}^{(\delta)}\left(s^{\prime}\right)$ are the unknown functions of our problem, and $\mathbf{K}\left(s, s^{\prime}\right)$ is the kernel of the integral equation. The integral is extended to the aperture ( $a p)$ of the junction (discontinuity) as shown in Fig. 1. The kernel of the integral equation is expressed through an infinite series, as

$$
\mathbf{K}\left(s, s^{\prime}\right)=\sum_{\delta=1}^{2} \sum_{m=N(\delta)+1}^{+\infty} Y_{m}^{(\delta)} \mathbf{h}_{m}^{(\delta) *}\left(s^{\prime}\right) \mathbf{h}_{m}^{(\delta)}(s) .
$$

To complete the rigorous network formulation, the elements of the generalized impedance matrix $Z$ are calculated as

$$
Z_{m, n}^{(\delta, \gamma)}=\int_{a p} \mathbf{M}_{n}^{(\gamma)}\left(s^{\prime}\right) \mathbf{h}_{m}^{(\delta) *}\left(s^{\prime}\right) d s^{\prime} .
$$

These equations can be interpreted in terms of the generalized network representation as shown in Fig. 2.

The MoM and the Galerking procedure [9] are usually employed to solve the integral equations in (2). To do that, we expand the unknown functions $\mathbf{M}_{n}^{(\gamma)}\left(s^{\prime}\right)$. Assuming that the index $r$ represents the smaller waveguide of the step, we use as basis functions the modes of the smaller waveguide $\mathbf{h}_{k}^{(r)}$

$$
M_{n}^{(\gamma)}\left(s^{\prime}\right)=\sum_{k=1}^{N_{b}} \alpha_{n, k}^{(\gamma)} \mathbf{h}_{k}^{(r)}\left(s^{\prime}\right) d s^{\prime}
$$

where $N_{b}$ determines the number of modes of the smaller waveguide that are used to expand the unknown functions. 
In other words, the numerical parameter $N_{b}$ fixes the number of basis and test functions in the MoM procedure.

As already mentioned, it is also important to review the transformation of the kernel first introduced in [10]. As shown in [10], the kernel can be rigorously split into a dynamic and static parts using Kummer's transformation [12], thus providing

$$
\begin{aligned}
\mathbf{K}\left(s, s^{\prime}\right)= & \mathbf{K}_{d}\left(s, s^{\prime}\right)+\mathbf{K}_{s}\left(s, s^{\prime}\right) \\
= & \sum_{\delta=1}^{2} \sum_{m=N(\delta)+1}^{N_{k_{d}}}\left(Y_{m}^{(\delta)}-\hat{Y}_{m}^{(\delta)}\right) \mathbf{h}_{m}^{(\delta) *}\left(s^{\prime}\right) \mathbf{h}_{m}^{(\delta)}(s) \\
& +\sum_{\delta=1}^{2} \sum_{m=N(\delta)+1}^{N_{k}} \hat{Y}_{m}^{(\delta)} \mathbf{h}_{m}^{(\delta) *}\left(s^{\prime}\right) \mathbf{h}_{m}^{(\delta)}(s)
\end{aligned}
$$

where $\hat{Y}_{m}^{(\delta)}$ represents the asymptotic behavior of the characteristic modal admittance when $k_{t, m}^{(\delta)} \gg k$.

The numerical parameters $N_{k_{d}}$ and $N_{k}$ represent the number of terms used in the dynamic and static kernel computation, respectively. The number of terms in the static kernel $\left(N_{k}\right)$ controls the upper limit in the summation of the frequencyindependent part of the Green's function, which describes the discontinuity at the junction of the two waveguides. In the cases in which some lower order localized modes do not satisfy the asymptotic approximation assumed in the static kernel, additional dynamic terms $N_{k_{d}}$ need to be computed.

This kernel formulation leads to the possibility of keeping to a minimum the number of accessible modes in the coupling matrix, and still having the important computational burden of the kernel independent of frequency.

In the regular convergence procedure, the minimum specific set of parameters that ensure a given degree of accuracy is identified by increasing manually their value until convergent results are obtained. As it is indicated in [5], the user should start by simulating the structure with increasing number of accessible modes (using a sufficiently large number of basis functions $N_{b}$ and terms in the kernel $N_{k}$ ). Once the required number of accessible modes is found, the process must be repeated for the number of basis functions, until the minimum number required for reaching convergence is identified. Finally, to fix the number of terms in the kernel, one has to simulate repeatedly the same structure with a sufficient number of terms and decrease this parameter progressively until the minimum required value is identified. This procedure requires a large number of repeated computations and is clearly very time-consuming.

To improve the situation, we propose in this contribution a new automatic strategy for the identification of the optimum set of computational parameters. It is important to note that the proposed method is possible thanks to the fact that, using the kernel formulation in (6), the number of accessible modes is not linked anymore to the convergence of the kernel. This is possible because of the fast converging dynamic summation that computes the lower order localized modes that do not satisfy the static kernel approximation. In this way, the number of accessible modes can now be fixed according to the physical structure of the device, independently of the kernel convergence issue. Using (6), this goal is possible, while the computational burden of the kernel remains frequency independent. In the following sections, we explain how the specific MEN convergence parameters can be efficiently adjusted automatically.

\section{A. Number of Accessible Modes}

The first parameter that needs to be adjusted is the number of accessible modes in each waveguide region of the junction (see Fig. 1). To establish an appropriate criterion for this selection, it is important to recall which modes are theoretically needed as accessible in the equivalent network (see Fig. 2). Naturally, all the modes that are propagating along the waveguide section must be taken into account as accessible modes, since they are responsible for the active energy exchange between the discontinuities in the structure. Furthermore, it is also known that the evanescent modes are storing energy near the discontinuities. Since in real structures many discontinuities are placed close to each other, some evanescent modes must also be considered as accessible ones, in order to achieve a complete characterization of the involved energy exchange process. In this representation, therefore, more modes than the propagating ones must, in general, be considered as accessible modes for each waveguide region in the equivalent network.

On this basis, the accessible modes in each region must be the propagating modes plus the evanescent ones that reach the next junction with enough energy. An automatic selection procedure must take into account that in waveguide sections with small lengths (such as thin coupling windows), we need to include more evanescent modes, since these modes are interacting with nearby discontinuities. On the other hand, in waveguide sections with large lengths (such as resonant cavities), only the first few propagating modes (with maybe one or two additional evanescent modes) are needed.

To cope with these very different situations, we propose a selection method based on fixing a power attenuation threshold in decibel $(\mathrm{dB})$ (that we call $\mathrm{dB}_{\mathrm{th}}$ ). The attenuation for each evanescent mode $\left(A t_{m}\right)$ along a distance $l$ can be calculated through its attenuation constant $\alpha_{m}$, as

$$
A t_{m}=\alpha_{m} \cdot l \text {. }
$$

According to this criterion, an evanescent mode will be considered to be accessible, if the total attenuation when it reaches the next discontinuity is smaller than the specified threshold. The number of accessible modes will then be given by all propagating modes plus the evanescent modes that satisfy the following condition (in $\mathrm{dB}$ )

$$
A t_{m} \leq \mathrm{dB}_{\mathrm{th}}
$$

which is imposed at the highest frequency in the analysis loop.

It is easy to see that this process automatically includes more evanescent modes in short waveguide sections, and less evanescent modes in long waveguide sections.

When analyzing a whole device, just a threshold $\mathrm{dB}_{\text {th }}$ needs to be fixed for the whole structure. The number of accessible modes required in each waveguide section is then adjusted automatically according to condition (8). 


\section{B. Number of Basis Functions}

As described in (5), the number of basis functions used for the MoM solution of the integral equation (2) is the number of vector mode functions of the smaller waveguide shown in Fig. 1. This also represents the number of test functions, since the Galerking procedure is normally used. In this case, we need to remember that the last mode we need to represent as excitation of our integral equation is the highest order accessible mode as indicated in (2). Once the threshold $\mathrm{dB}_{\text {th }}$ is set, this mode corresponds to the highest order mode that satisfies the condition in (8). From now on, the cut-off wavenumber of this mode is referred to as $k c_{\max }$. In the proposed procedure, all the modes of the smaller waveguide with a cut-off wavenumber smaller or equal to this value (times an accuracy factor $F_{b}$ ) are automatically selected as basis functions. This condition can be written as

$$
N_{b} \rightarrow k c_{m} \leq F_{b} \cdot k c_{\max }
$$

where $F_{b}$ is considered as a factor of precision. It is clear that if the factor $F_{b}$ is increased, more modes will satisfy the condition in (9). This leads to a higher number of basis functions to be used in the MoM solution.

The criterion to identify the correct $F_{b}$ value is based on the numerical convergence of the value of the last element of the impedance submatrix that represents the autointeraction of the smaller waveguide. According to (4), this last element is written as $Z_{N(r), N(r)}^{(r, r)}$. In this paper, we propose to use the relative error in the computation of this element of the impedance matrix to identify the value of the $F_{b}$ factor, evaluated at the highest frequency in the analysis loop. The computational effort of this evaluation is really small in comparison to what is needed to adjust manually this parameter. Once the $\mathrm{dB}_{\text {th }}$ value is fixed by the user, the selection of $F_{b}$ is accomplished automatically, without any user intervention, to ensure convergence.

\section{Number of Terms in the Kernel}

According to (6), the $N_{k}$-parameter refers to the number of terms that is summed in the static kernel of the integral equation. In other words, it represents the number of terms in the frequency-independent summation of the Green's functions. As for the basis functions, the kernel convergence depends on the geometry of the step and also on the excitation of the integral equation. In this respect, using the same cut-off wavenumber of the last accessible mode $k c_{\max }$, the number of modes in the kernel $\left(N_{k}\right)$ will be taken as those modes of the bigger waveguide with their cut-off wavenumbers $k c_{n}$ lower than the selected $k c_{\text {max }}$ times an accuracy $F_{k}$ factor.

This condition can be written as

$$
N_{k} \rightarrow k c_{n} \leq F_{k} \cdot k c_{\text {max }}
$$

where $F_{k}$ is, as before, the precision factor. The adjustment of this factor is going to be carried out according to the convergence study on the element $Z_{N(r), N(r)}^{(r, r)}$, which is accomplished automatically.

A convenient option is to select the same factor $F_{b}=F_{k}=F$ to fix both the precision associated with the

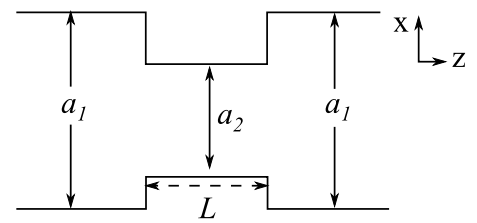

Fig. 3. Inductive iris composed of two steps in waveguide technology. The dimensions are $a_{1}=19.1 \mathrm{~mm}$ and $a_{2}=9.0203 \mathrm{~mm}$, and the height is $b=8.2 \mathrm{~mm}$.

number of basis functions and to the number of terms in the kernel. To simplify the convergence procedure, the same $F$ value is indeed used in this contribution. Therefore, if the $F$ factor is increased, more basis functions and terms in the kernel will be used in the numerical solution of the problem.

When analyzing a complex device, the parameter $F$, which adjusts the specific parameters $N_{b}$ and $N_{k}$, is automatically computed for each discontinuity, to assure convergence, and therefore, the user is released of that responsibility. This results in a significant reduction of the computational effort together with an improved user simulation experience.

The complete picture is finalized with the calculation of the dynamic part of the kernel as shown in (6). This parameter is related to the Taylor expansion of the static approximation, and converges with a few terms $\left(N_{k_{d}}\right)$. The convergence of this last term was extensively treated in [10]. On this basis, we have taken a fix number $N_{k_{d}}=10$ for all the practical examples considered in this paper.

It is important to remark that the static part of the kernel only contains the higher order modes that satisfy the static approximation. Thanks to the dynamic part, the localized modes that do not satisfy the static approximation are no longer considered as accessible modes (as it is done in the original formulation), but are considered in the dynamic kernel as localized.

\section{NumericAl Results AND Applications}

In this section, the proposed convergence method is applied in an MEN implementation to analyze some inductive waveguide structures. First of all, an iris between two waveguides will be analyzed using the new mechanism to show how the convergence parameters are adjusted according to different lengths. Then, some practical inductive filters are also studied using the new strategy, to show the performance improvement achieved in the analysis of real waveguide structures.

\section{A. Study on Simple Waveguide Iris}

In this section, a simple iris between two waveguides shown in Fig. 3 is analyzed, using the MEN formulation together with the convergence method proposed in this contribution. To show the behavior of the described technique according to the length of the iris, the structure in Fig. 3 is studied for different values of $L$.

At the input and output waveguides, only the propagating modes need to be considered as accessible modes. However, 
TABLE I

Number of Accessible Modes Adjusted By the New Method ACCORding to THE LENGTH $L$ AND THE THRESHOLD $\mathrm{dB}_{\text {th }}$

\begin{tabular}{|l|c|c|c|}
\hline$d B_{t h}$ & $L=0.5 \mathrm{~mm}$ & $L=1 \mathrm{~mm}$ & $L=5 \mathrm{~mm}$ \\
\hline \hline $0 \mathrm{~dB}$ & $3,1,3$ & $3,1,3$ & $3,1,3$ \\
$10 \mathrm{~dB}$ & $3,6,3$ & $3,3,3$ & $3,1,3$ \\
$20 \mathrm{~dB}$ & $3,13,3$ & $3,6,3$ & $3,2,3$ \\
$30 \mathrm{~dB}$ & $3,19,3$ & $3,10,3$ & $3,2,3$ \\
\hline
\end{tabular}

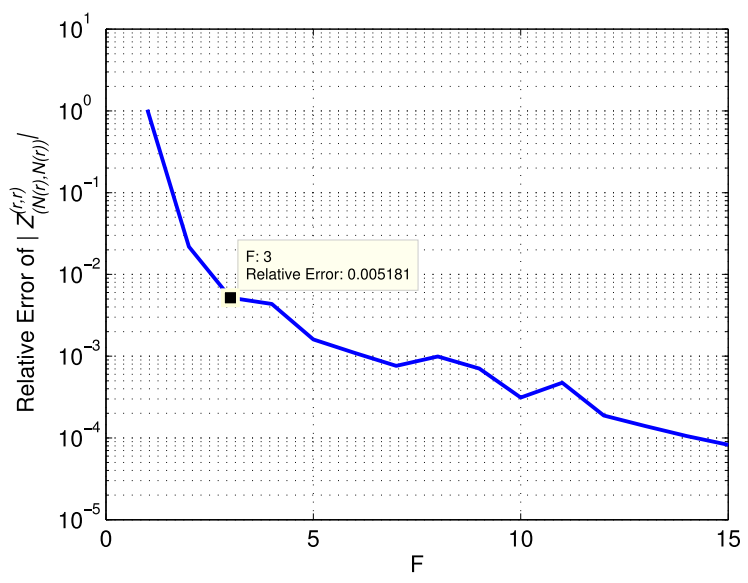

Fig. 4. Relative convergence error of the element $Z_{N(r), N(r)}^{(r, r)}$ as a function of the accuracy $F$ factor for the first step in Fig. 3.

in the iris, the propagating plus some evanescent modes will need to be accessible.

With the new technique, the authors are suggesting not to fix directly the number of accessible modes but a global threshold $\mathrm{dB}_{\mathrm{th}}$. With this power attenuation threshold, the number of accessible modes needed to satisfy the specified attenuation condition level in each region will automatically be fixed.

In this context, in Table I, the number of accessible modes $(N)$ that the proposed method fixes for each medium and for different values of $\mathrm{dB}_{\mathrm{th}}$ is reported.

As it is noticed, for $\mathrm{dB}_{\mathrm{th}}=0 \mathrm{~dB}$, since no attenuation is specified, only the propagating modes are accessible in all three regions. Moreover, when the attenuation threshold is increased, more evanescent modes are considered to be accessible in the iris. Also, the number of accessible modes increases for thinner irises, as it is required to represent the stronger energy exchange between nearby discontinuities.

Once this parameter has been chosen by the user, the factor $F$ is automatically adjusted, and consequently also the number of basis functions and the number of terms in the static kernel. For the problems treated in this paper, we have observed that relative errors in the order of $10^{-3}$ during the calculation of the $F$ factors lead to good accuracy. Thus, for each discontinuity, the factor $F$ is selected as the one which gives a relative convergence error in the order of $10^{-3}$ for the element $Z_{N(r), N(r)}^{(r, r)}$ of the generalized impedance matrix.

In Fig. 4, we show this relative error as a function of increasing the accuracy $(F)$ parameter for the first step of Fig. 3. In this test, we have fixed $\mathrm{dB}_{\text {th }}=10 \mathrm{~dB}$ and $L=0.5 \mathrm{~mm}$. As it is shown, for this step, a factor $F=3$ is needed to obtain an error in the order of $10^{-3}$.
TABLE II

Parameters Adjusted by the Method Using $\mathrm{dB}_{\mathrm{th}}=10 \mathrm{~dB}$ AND $\mathrm{dB}_{\text {th }}=20 \mathrm{~dB}$ FOR DIFFERENT LENGTHS $L$

\begin{tabular}{|c|c|c|c|}
\hline & $L=0.5 \mathrm{~mm}$ & $L=1 \mathrm{~mm}$ & $L=5 \mathrm{~mm}$ \\
\hline \hline \multirow{5}{*}{$10 \mathrm{~dB}$} & $N=(3,6,3)$ & $N=(3,3,3)$ & $N=(3,1,3)$ \\
& $F=3$ & $F=4$ & $F=9$ \\
& $N_{b}=17$ & $N_{b}=12$ & $N_{b}=12$ \\
& $N_{k}=38$ & $N_{k}=25$ & $N_{k}=27$ \\
& $\epsilon=3.23 \cdot 10^{-4}$ & $\epsilon=6.65 \cdot 10^{-4}$ & $\epsilon=3.10 \cdot 10^{-4}$ \\
\hline \multirow{3}{*}{$20 \mathrm{~dB}$} & $N=(3,13,3)$ & $N=(3,6,3)$ & $N=(3,2,3)$ \\
& $F=2$ & $F=3$ & $F=6$ \\
& $N_{b}=26$ & $N_{b}=17$ & $N_{b}=12$ \\
& $N_{k}=55$ & $N_{k}=38$ & $N_{k}=25$ \\
& $\epsilon=4.89 \cdot 10^{-4}$ & $\epsilon=3.14 \cdot 10^{-4}$ & $\epsilon=3.23 \cdot 10^{-4}$ \\
\hline
\end{tabular}

In Table II, the automatically selected parameters $F, N_{b}$, and $N_{k}$ are presented for each considered iris length. The absolute convergence errors $(\epsilon)$ achieved with respect to the convergent response are also included. The convergent response is obtained by selecting arbitrary large numerical parameters (in the examples treated in this paper 20 accessible modes, 200 basis functions, and 2000 terms in the kernel). Note that this time the absolute error is given instead of the relative error. This is because the response contains poles and zeros, which make inaccurate the calculation of the relative errors at the corresponding frequencies.

In this case, we also consider two different threshold levels $\mathrm{dB}_{\mathrm{th}}, 10$ and $20 \mathrm{~dB}$, to see how the factor $F$ is adjusted. For completeness, the number of accessible modes $(N)$ in the three regions are also given.

It can be seen that, for irises that require a smaller number of accessible modes, a bigger $F$ value is selected by the algorithm, and this effectively preserves the accuracy of the computations.

As it is noticed, the proposed selecting mechanism simplifies the current manual convergence study since a physical parameter, the threshold $\mathrm{dB}_{\mathrm{th}}$, is just required to be fixed according to the desired accuracy level. Then, the factor of precision $F$ is automatically adjusted, and finally, the number of basis functions and terms in the kernel are automatically selected according to the conditions (9) and (10), to provide a convergent response.

Another important fact is that until now, for the sake of simplification, MEN users used to select the same parameters for all the discontinuities within a structure (global parameters). This can oversize the less restrictive discontinuities until the others reach convergence. With this new convergence mechanism, the user can still select global parameters, that is, the same threshold $\mathrm{dB}_{\text {th }}$ for all the discontinuities. However, with the new algorithm this implies to use different specific parameters (number of accessible modes, number of basis functions, and terms in the kernel) in each discontinuity according to their specific geometries. As a consequence, all discontinuities have different numerical parameters better suited to their specific needs, still using intuitive and global parameters for the user. 


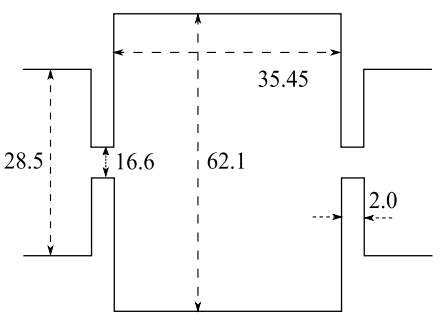

Fig. 5. Single dual-mode inductive cavity under study (dimensions in $\mathrm{mm}$ ).

TABLE III

Convergence Parameters Adjusted Automatically BY THE Method Using $\mathrm{dB}_{\text {th }}=10 \mathrm{~dB}$

\begin{tabular}{|l|c|c|c|c|}
\hline & Step 1 & Step 2 & Step 3 & Step 4 \\
\hline \hline$N$ & $(1,3)$ & $(3,3)$ & $(3,3)$ & $(3,1)$ \\
\hline$F$ & 5 & 4 & 4 & 5 \\
$N_{b} / N_{k}$ & $15 / 25$ & $12 / 44$ & $12 / 44$ & $15 / 25$ \\
\hline
\end{tabular}

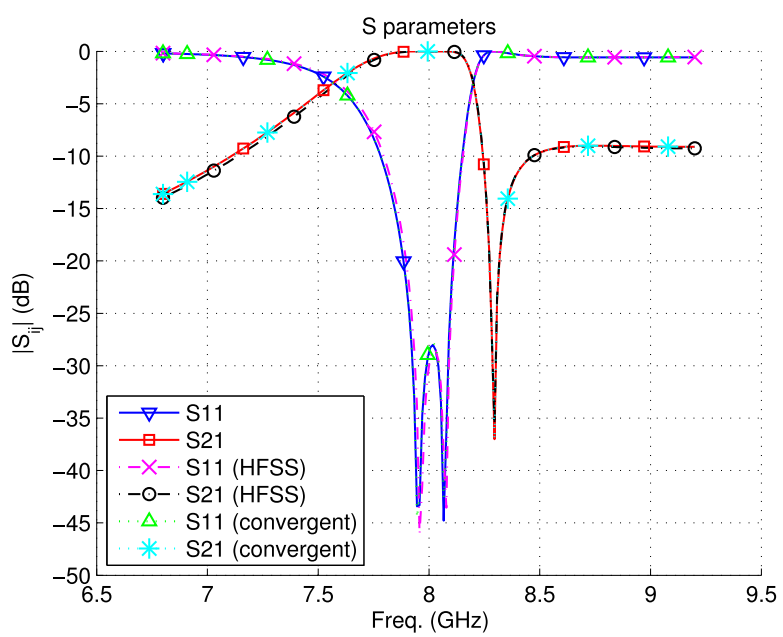

Fig. 6. $S$-parameters for the dual-mode cavity filter in Fig. 5 using the parameters adjusted by the proposed method (see Table III) and compared to the convergent performance using MEN formulation and HFSS software tool.

\section{B. Practical Filtering Structures}

In this section, the proposed convergence method is implemented in the MEN technique to analyze some practical inductive filters. In these cases, the waveguide filters are composed of short and large length sections, mixed together. Thus, the advantage of being able to use a global parameter for the whole structure (while the actual numerical parameters are adjusted differently for each discontinuity) is significant in these examples.

The first example is proposed in [13], and consists of a single dual-mode inductive cavity as the one shown in Fig. 5.

For a threshold $\mathrm{dB}_{\text {th }}=10 \mathrm{~dB}$, the factors $F$ and the specific parameters $N, N_{b}$, and $N_{k}$, automatically selected for each discontinuity, are shown in Table III.

The $S$-parameters simulated using the specific parameters in Table III are compared in Fig. 6 to the convergent response for 20 accessible modes, 200 basis functions, and 2000 terms in the kernel and to HFSS simulation results.

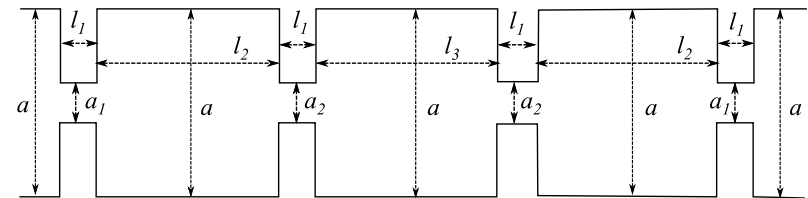

Fig. 7. Three-pole filter under study. The dimensions are $a=19.1 \mathrm{~mm}$, $a_{1}=9.0203 \mathrm{~mm}, a_{2}=5.1702 \mathrm{~mm}$, and the height is $b=8.2 \mathrm{~mm}$. The lengths are $l_{1}=1 \mathrm{~mm}, l_{2}=21.6581 \mathrm{~mm}$, and $l_{3}=23.1495 \mathrm{~mm}$.

TABLE IV

Convergence Parameters Adjusted Automatically B Y THE METhod Using $\mathrm{dB}_{\text {th }}=20 \mathrm{~dB}$

\begin{tabular}{|l|c|c|c|c|}
\hline & Steps 1 / 8 & Steps 2 / 7 & Steps 3 / 6 & Steps 4 / 5 \\
\hline \hline$N$ & $(3,6)$ & $(6,1)$ & $(1,3)$ & $(3,1)$ \\
\hline$F$ & 3 & 3 & 4 & 4 \\
$N_{b} / N_{k}$ & $17 / 38$ & $17 / 38$ & $12 / 44$ & $12 / 44$ \\
\hline
\end{tabular}

The absolute convergence error is of $4.47 \cdot 10^{-4}$. As we can see, a good agreement between the convergent response and the $S$-parameters obtained with the proposed method are achieved. In addition, a great reduction in required simulation parameters is noticed, which leads to a reduction in the computational effort. Using an Intel Xeon E5-2620 v3 at $2.40 \mathrm{GHz}$ with $32 \mathrm{~GB}$ of RAM, the computational time per frequency point required to achieve the HFSS results in Fig. 6 is $1 \mathrm{~min}$ and $1 \mathrm{~s}$, while for the proposed MEN implementation it is of $1.14 \mathrm{~s}$. It is important to remember that our software tool is specifically designed to analyze this type of waveguide structures in contrast to HFSS, which is a more generic analysis tool.

The second example consists of a three-pole in-line inductive waveguide filter (see Fig. 7). For a global threshold $\mathrm{dB}_{\mathrm{th}}=20 \mathrm{~dB}$, the selected parameters of each discontinuity are shown in Table IV.

It can be observed that the proposed technique increases the value of $F$ when the number of accessible modes decreases. This tends to keep a constant numerical accuracy along all the discontinuities of the structure.

The resulting $S$-parameters, compared to the convergent response for 20 accessible modes, 200 basis functions, and 2000 terms in the kernel and to results obtained with the commercial software tool HFSS, are shown in Fig. 8. In this case, we also include the performance for a lower threshold, $\mathrm{dB}_{\text {th }}=10 \mathrm{~dB}$, to show how the accuracy level can be controlled through this parameter.

In this example, for $\mathrm{dB}_{\text {th }}=20 \mathrm{~dB}$, the absolute convergence error is of $6.33 \cdot 10^{-4}$, indicating good numerical accuracy. Moreover, a resulting reduction of the parameters in the numerical method is effectively achieved. The computational time per frequency point required to achieve the HFSS results in Fig. 8 is $15 \mathrm{~min}$ and $13 \mathrm{~s}$, while for the proposed MEN implementation it is of $1.49 \mathrm{~s}$.

The last example is proposed in [13] and consists of a four-pole dual-mode inductive filter shown in Fig. 9. The dimensions are reported in Table $\mathrm{V}$.

For a threshold $\mathrm{dB}_{\text {th }}=20 \mathrm{~dB}$, the factors $F$ and the adjusted parameters for each discontinuity, are shown in Table VI.

The resulting $S$-parameters, compared to the convergent response for 20 accessible modes, 200 basis functions, and 


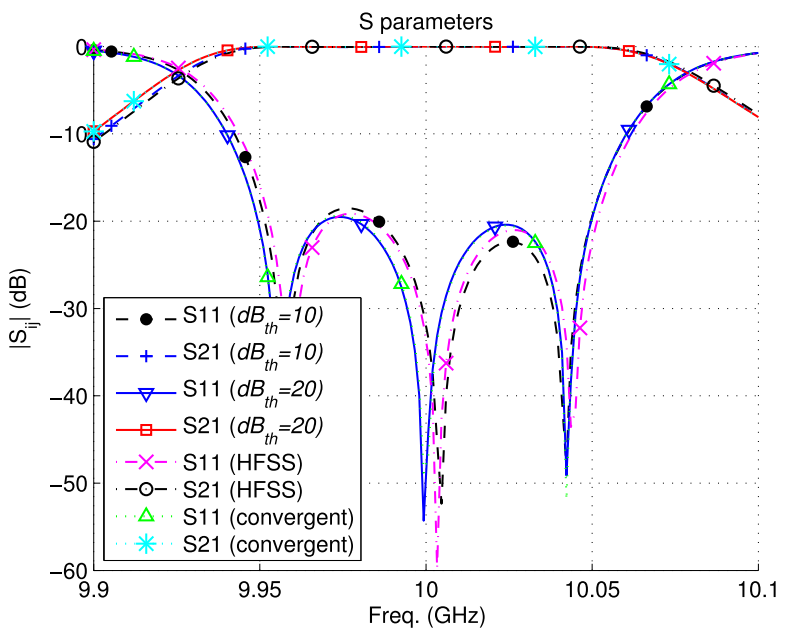

Fig. 8. $S$-parameter for the three-pole filter in Fig. 7 using the parameters adjusted by the proposed method for $\mathrm{dB}_{\text {th }}=10 \mathrm{~dB}$ and $\mathrm{dB}_{\text {th }}=20 \mathrm{~dB}$ (see Table IV) and compared to the convergent performance using MEN formulation and HFSS software tool.

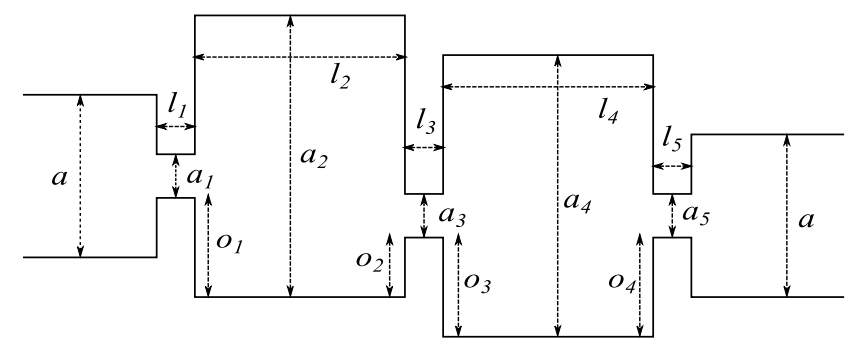

Fig. 9. Four-pole dual-mode filter in the Ku-band. The dimensions are reported in Table $\mathrm{V}$ and the height is $b=9.525 \mathrm{~mm}$.

TABLE V

Dimensions of the Four-Pole Dual-Mode Filter in Fig. 9

\begin{tabular}{|l|l|l|}
\hline$a=19.05 \mathrm{~mm}$ & & \\
$a_{1}=10.931 \mathrm{~mm}$ & $l_{1}=3 \mathrm{~mm}$ & $o_{1}=1 \mathrm{~mm}$ \\
$a_{2}=30.077 \mathrm{~mm}$ & $l_{2}=28.306 \mathrm{~mm}$ & $o_{2}=1 \mathrm{~mm}$ \\
$a_{3}=10.782 \mathrm{~mm}$ & $l_{3}=12.481 \mathrm{~mm}$ & $o_{3}=0.87 \mathrm{~mm}$ \\
$a_{4}=29.567 \mathrm{~mm}$ & $l_{4}=28.852 \mathrm{~mm}$ & $o_{4}=0.87 \mathrm{~mm}$ \\
$a_{5}=10.596 \mathrm{~mm}$ & $l_{5}=3 \mathrm{~mm}$ & \\
\hline
\end{tabular}

2000 terms in the kernel and to HFSS simulation results, are shown in Fig. 10.

The absolute convergence error in this case is of $8.08 \cdot 10^{-4}$, showing again the effectiveness of the proposed technique. The computational time required for each discrete frequency point to achieve the HFSS results reported in Fig. 8 is $11 \mathrm{~min}$ and $33 \mathrm{~s}$, while the time for the proposed MEN implementation is of $1.28 \mathrm{~s}$.

This example is appropriate for an in-depth study on the importance of adjusting different $F$ factors for each discontinuity. As we can see in Table VI, if the factor $F$ is selected to be the same, it should be the biggest to assure convergence in all the discontinuities. In this case, it would be $F=10$ to assure convergence in steps 3 and 4 . This would imply that $N_{b}$ and $N_{k}$ in rest of the discontinuities would

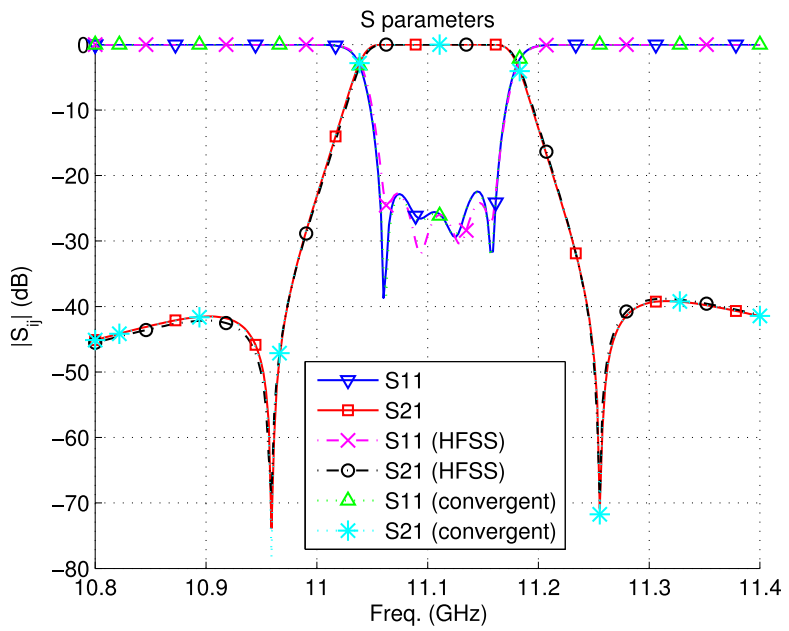

Fig. 10. $S$-parameter for the four-pole dual-mode filter in Fig. 9 using the parameters adjusted by the proposed method (see Table VI) and compared to the convergent performance using MEN formulation and HFSS software tool.

TABLE VI

Convergence Parameters Adjusted Automatically By THE METhod Using dB $\mathrm{dh}_{\text {th }}=20 \mathrm{~dB}$

\begin{tabular}{|l|c|c|c|c|c|c|}
\hline & Step 1 & Step 2 & Step 3 & Step 4 & Step 5 & Step 6 \\
\hline \hline$N$ & $(1,2)$ & $(2,2)$ & $(2,1)$ & $(1,2)$ & $(2,2)$ & $(2,1)$ \\
\hline$F$ & 5 & 5 & 10 & 10 & 5 & 5 \\
$N_{b} / N_{k}$ & $10 / 17$ & $10 / 27$ & $10 / 27$ & $10 / 27$ & $10 / 27$ & $10 / 17$ \\
\hline
\end{tabular}

TABLE VII

TOTAL COMPUTATIONAL TIME FOR DIFFERENT NUMERICAL PARAMETERS IN THE MEN IMPLEMENTATION USING 250 FREQUENCY POINTS

\begin{tabular}{|c|c|c|c|}
\hline$N$ & $N_{b}$ & $N_{k}$ & Time $(\mathrm{s})$ \\
\hline \hline 2 & 20 & 55 & 2.64 \\
\hline 5 & 30 & 300 & 4.71 \\
\hline 10 & 50 & 500 & 12.75 \\
\hline 15 & 100 & 1000 & 31.85 \\
\hline
\end{tabular}

also be increased. In other words, the parameters for some discontinuities would be increased at the expense of reaching convergence in the most limiting discontinuities. Since this increment is unnecessary, this would result in an inefficient oversizing of the convergence parameters. This problem is avoided with the strategy proposed in this paper, since the algorithm automatically adjusts different $F$ factors for each discontinuity.

As these examples show, the new mechanism for fixing the convergence parameters in MEN implementations gives accurate responses along with a more efficient computation (thanks to the achieved reduction in the number of the involved numerical parameters).

In this respect, in Table VII, the computational times required by different numerical parameters in the MEN implementation for analyzing this last geometry using 250 frequency points are reported.

Table VII shows that increasing the numerical MEN parameters affects negatively the computational effort. In comparison, the analysis of the same filter using the technique described in this paper is only $1.89 \mathrm{~s}$. Thus, Table VII shows 
that an important reduction in computational time can be obtained, if the numerical parameters of the MEN are kept to the strict minimum required, as calculated by the new strategy proposed in this paper.

\section{CONCLUSION}

A simple and elegant strategy to choose efficiently the numerical parameters needed to reach convergence in the MEN formulation has been proposed.

With the proposed mechanism, only the parameter $\mathrm{dB}_{\text {th }}$ needs to be fixed by the software user. The chosen $\mathrm{dB}_{\text {th }}$ value will then affect the accuracy of the obtained results. The other computational parameter needed, namely $F$, is automatically adjusted according to the convergence of the last element of the multimode impedance matrix. This leads to the correct adjustment of all specific computational parameters (namely, $N, N_{b}$, and $N_{k}$ ), different for each discontinuity, by just fixing the same attenuation threshold for the whole structure.

The first advantage is related to the fact that, for each discontinuity, the actual numerical parameters are different according to their specific convergence needs, thus the oversizing of discontinuities is avoided. The second advantage is on user level. The proposed procedure implies a more intuitive way of selecting just a parameter that controls the accuracy of the calculations. This also leads to a huge saving in computational effort, as compared to the convergence study currently required in commercially available MEN implementations. In addition to theory, numerical results achieved using the proposed mechanism have also been provided, indicating that the new procedure leads to more efficient MEN calculations, while producing an excellent numerical accuracy.

\section{REFERENCES}

[1] M. Salazar-Palma, T. K. Sarkar, L.-E. Garcia-Costillo, T. Roy, and A. Djordjevic, Iterative and Self-Adaptive Finite-Elements in Electromagnetic Modeling. Norwood, MA, USA: Artech House, 1998.

[2] A. F. Peterson, S. L. Ray, and R. Mittra, FiniteDifference TimeDomain Methods on Orthogonal Meshes. New York, NY, USA: Wiley, 1998, pp. $495-523$.

[3] M. Guglielmi and C. Newport, "Rigorous, multimode equivalent network representation of inductive discontinuities," IEEE Trans. Microw. Theory Techn., vol. 38, no. 11, pp. 1651-1659, Nov. 1990.

[4] P. Arcioni, M. Bressan, G. Conciauro, and L. Perregrini, "Generalized Y-matrix of arbitrary H-plane waveguide junctions by the BI-RME method," in IEEE MTT-S Int. Microw. Symp. Dig., Denver, CO, USA, Jun. 1997, pp. 211-214.

[5] B. Casali, M. Ghilardi, and J. G. Raga, "FEST3D: A multimode CAD tool for passive mm-wave and microwave circuit modelling," in Proc. Prog. Electromagn. Res. Symp., Pisa, Italy, Mar. 2004, pp. 193-196.

[6] T. Itoh, Numerical Techniques for Microwave and Millimeter-Wave Passive Structures. Hoboken, NJ, USA: Wiley, 1989.

[7] G. Conciauro, M. Guglielmi, and R. Sorrentino, Advanced Modal Analysis: CAD Techniques for Waveguide Components and Filters. New York, NY, USA: Wiley, 1999.

[8] A. A. Melcón and M. Guglielmi, "Multimode network representation of two dimensional steps in rectangular waveguides," in Proc. 24th Eur. Microw. Conf., Sep. 1994, pp. 1943-1948.

[9] R. F. Harrington, Field Computation by Moment Methods. Hoboken, NJ, USA: Wiley, 1993.

[10] C. G. Molina, F. Q. Pereira, A. A. Melcon, V. B. Esbert, and M. Guglielmi, "Efficient formulation of multimode equivalent networks for 2-D waveguide steps through Kummers transformation," in IEEE MTT-S Int. Microw. Symp. Dig., Numer. Electromagn. Multiphys. Modeling Optim. RF, Microw., Terahertz Appl. (NEMO), May 2017, pp. $149-151$.
[11] N. Marcuvitz, Waveguide Handbook (M.I.T. Radiation Laboratory Series), vol. 10. New York, NY, USA: McGraw-Hill, 1951.

[12] A. L. Fructos, R. R. Boix, F. Mesa, and F. Medina, "An efficient approach for the computation of 2-D green's functions with 1-D and 2-D periodicities in homogeneous media," IEEE Trans. Antennas Propag., vol. 56, no. 12, pp. 3733-3742, Dec. 2008

[13] M. Guglielmi, P. Jarry, E. Kerherve, O. Roquebrun, and D. Schmitt, "A new family of all-inductive dual-mode filters," IEEE Trans. Microw. Theory Techn., vol. 49, no. 10, pp. 1764-1769, Oct. 2001.

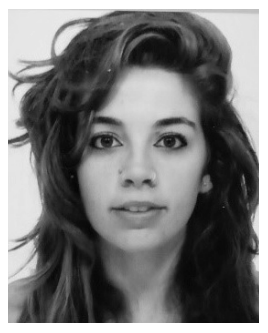

Celia Gómez Molina was born in Murcia, Spain, in 1992. She received the bachelor's degree in telecommunication systems and master's degree in telecommunication engineering from the Technical University of Cartagena (UPCT), Murcia, in 2014 and 2016, respectively.

In 2016, she joined the Department of Information Technologies and Communications, UPCT, as a Research Student, where she is currently developing her teaching and research activities. She is involved in the development of analytical and numerical tools for network representations of waveguide and planar discontinuities. Her current research interest includes the design of microwave filters.

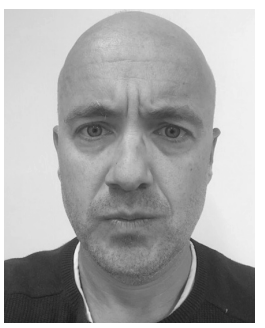

Fernando Quesada Pereira (S'05-M'08) was born in Murcia, Spain, in 1974. He received the Telecommunications Engineer degree from the Technical University of Valencia (UPV), Valencia, Spain, in 2000, and the Ph.D. degree from the Technical University of Cartagena (UPCT), Murcia, in 2007.

In 1999, he joined the Radiocommunications Department, UPV, as a Research Assistant, where he was involved in the development of numerical methods for the analysis of anechoic chambers and tag antennas. In 2001, he joined the Communications and Information Technologies Department, UPCT, as a Research Assistant, and then as an Assistant Professor. In 2005, he was a Visiting Scientist with the University of Pavia, Pavia, Italy. In 2009, he was an Invited Researcher with UPV. In 2011, he became an Associate Professor with UPCT. His current research interest includes integral equation numerical methods for the analysis of antennas and microwave devices.

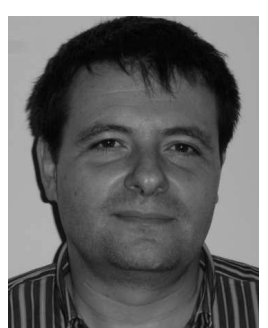

Alejandro Álvarez Melcón (M'99-SM'07) was born in Madrid, Spain, in 1965. He received the Telecommunications Engineer degree from the Technical University of Madrid (UPM), Madrid, in 1991, and the Ph.D. degree in electrical engineering from the Swiss Federal Institute of Technology, Lausanne, Switzerland, in 1998

In 1988, he joined the Signal, Systems and Radiocommunications Department, UPM, as a Research Student, where he was involved in the design, testing, and measurement of broadband spiral antennas for electromagnetic measurements support equipment. From 1991 to 1993, he was with the Radio Frequency Systems Division, European Space Agency/European Space Research and Technology Center (ESA/ESTEC), Noordwijk, The Netherlands, where he was involved in the development of analytical and numerical tools for the study of waveguide discontinuities, planar transmission lines, and microwave filters. From 1993 to 1995, he was with the Space Division, Industry Alcatel Espacio, Madrid, and also with ESA, where he was involved in the several ESA/ESTEC contracts. From 1995 to 1999, he was with the Swiss Federal Institute of Technology, École Polytechnique Fédérale de Lausanne, Lausanne, where he was involved in the field of microstrip antennas and printed circuits for space applications. In 2000, he joined the Technical University of Cartagena, Cartagena, Spain, where he is currently developing his teaching and research activities.

Dr. Álvarez Melcón was a recipient of the Journée Internationales de Nice Sur les Antennes (JINA) Best Paper Award for the best contribution to the JINA'98 International Symposium on Antennas, and the Colegio Oficial de Ingenieros de Telecomunicación (COIT/AEIT) Award for the best Ph.D. dissertation in basic information and communication technologies. 


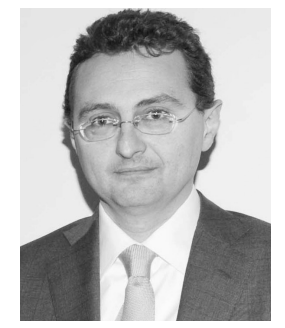

Vicente E. Boria (S'91-A'99-SM'02-F'18) was born in Valencia, Spain, in 1970. He received the Ingeniero de Telecomunicación degree (with FirstClass Hons.) and Doctor Ingeniero de Telecomunicación degree from the Universidad Politécnica de Valencia, Valencia, in 1993 and 1997, respectively.

In 1993, he joined the Departamento de Comunicaciones, Universidad Politécnica de Valencia. In 1995 and 1996, he was a Spanish Trainee with the European Space Research and Technology Center, European Space Agency (ESTEC-ESA), Noordwijk, The Netherlands, where he was involved in the EM analysis and design of passive waveguide devices. Since 2003, he has been a Full Professor with the Universidad Politécnica de Valencia. He has authored or co-authored 10 chapters in technical textbooks, 160 papers in refereed international technical journals, and over 200 papers in international conference proceedings. His current research interests include the analysis and automated design of passive components, left-handed and periodic structures, as well as on the simulation and measurement of power effects in passive waveguide systems.

Dr. Boria has been a member of the IEEE Microwave Theory and Techniques Society (IEEE MTT-S) and the IEEE Antennas and Propagation Society since 1992. He is a member of the Editorial Boards of the IEEE Transactions on Microwave Theory and Techniques, IEEE Microwave And Wireless Components LetTers, Proceeding of the IET (Microwaves, Antennas and Propagation), IET Electronics Letters and Radio Science. He was an Associate Editor of IEEE MICROWAVE AND WIRELESS COMPONENTS LETTERS and IET Electronics Letters. He is also a member of the Technical Committees of the IEEE MTT-S International Microwave Symposium and of the European Microwave Conference.

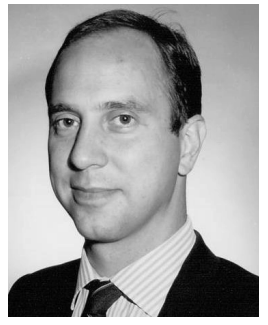

Marco Guglielmi (S'79-M'81-SM'97-F'13) was born in Rome, Italy, in 1954. He received the Laurea degree in ingegneria elettronica from the University of Rome "La Sapienza," Rome, in 1979, the M.S. degree in electrical engineering from the University of Bridgeport, Bridgeport, CT, USA, in 1982, and the Ph.D. degree in electrophysics from the Polytechnic University, Brooklyn, NY, USA, in 1986.

He was an Academic Associate from 1984 to 1986

and an Assistant Professor from 1986 to 1988 with the Polytechnic University. From 1988 to 1989 , he was an Assistant Professor with the New Jersey Institute of Technology, Newark, NJ, USA. In 1989, he joined the European Space Agency, as a Senior Microwave Engineer with the RF System Division, European Space Research and Technology Center (ESTEC), Noordwijk, The Netherlands, where he was in charge of the development of microwave filters and electromagnetic simulation tools. In 2001, he was the Head of the Technology Strategy Section, ESTEC, where he was involved in the development of management processes and tools for the formulation of a European strategy for space technology research and development. In 2014, he was retired from the European Space Agency and is currently an Invited Senior Researcher with the Polytechnic University of Valencia, Valencia, Spain.

Dr. Guglielmi was a recipient of the Fulbright Scholarship in Rome and the Halsey International Scholarship Program from the University of Bridgeport in 1981 . 